\title{
A novel APC mutation identified in a large Chinese family with familial adenomatous polyposis and a brief literature review
}

\author{
MINGHUI PANG ${ }^{1 *}$, YIJUN LIU ${ }^{2 *}$, XIAOLIN HOU $^{1 *}$, JIALIANG YANG $^{3}$, XUELAI HE $^{1}$, NENGYI HOU $^{1}$, \\ PEIXI LIU ${ }^{5}$, LUO LIANG ${ }^{1}$, JUNWEN FU ${ }^{1}$, KANG WANG $^{1}$, ZIMENG YE ${ }^{3}$ and BO GONG ${ }^{3,4}$ \\ ${ }^{1}$ Department of Gastrointestinal Surgery, Sichuan Academy of Medical Sciences and Sichuan Provincial People's Hospital, \\ School of Medicine, University of Electronic Science and Technology of China, Chengdu, Sichuan 610072; \\ ${ }^{2}$ State Key Laboratory of Oncology in South China, Collaborative Innovation Center for Cancer Medicine, Department of \\ Clinical Laboratory Medicine, Sun Yat-Sen University Cancer Center, Guangzhou, Guangdong 510000; ${ }^{3}$ Sichuan Provincial \\ Key Laboratory for Human Disease Gene Study, Sichuan Academy of Medical Sciences and Sichuan Provincial People's Hospital, \\ School of Medicine, University of Electronic Science and Technology of China, Chengdu, Sichuan 610072; ${ }^{4}$ Institute of \\ Chengdu Biology, Sichuan Translational Medicine Hospital, Chinese Academy of Sciences, Chengdu, Sichuan 610000; \\ ${ }^{5}$ Department of Gastroenterology, Sichuan Academy of Medical Sciences and Sichuan Provincial People's Hospital, \\ School of Medicine, University of Electronic Science and Technology of China, Chengdu, Sichuan 610072, P.R. China
}

Received January 12, 2018; Accepted May 9, 2018

DOI: $10.3892 / \mathrm{mmr} .2018 .9130$

\begin{abstract}
Familial adenomatous polyposis (FAP), an autosomal dominant disease, is a colon cancer predisposition syndrome that manifests as a large number of adenomatous polyps. Mutations in the Adenomatous polyposis coli (APC) gene are responsible for the majority of cases of FAP. The purpose of the present study was to report the clinical features of a Chinese family with FAP and screen for novel mutations using the targeted next-generation sequencing technology. Among the 29 family members, 12 were diagnosed of FAP. Based on an established filtering strategy and data analyses, along with confirmation by Sanger sequencing and co-segregation, a novel frameshift mutation c.1317delA (p.Ala440LeufsTer14) in exon 10 of the $A P C$ gene was identified. To the best of our knowledge, this mutation has not been reported prior to the present study. In addition, it was correlated with extra-colonic phenotypes featuring duodenal polyposis and sebaceous cysts in this family. This novel frameshift mutation causing FAP not only expands the germline mutation spectrum of the
\end{abstract}

Correspondence to: Dr Bo Gong or Dr Zimeng Ye, Sichuan Provincial Key Laboratory for Human Disease Gene Study, Sichuan Academy of Medical Sciences and Sichuan Provincial People's Hospital, School of Medicine, University of Electronic Science and Technology of China, 32 Road West 2, The First Ring Road, Chengdu, Sichuan 610072, P.R. China

E-mail: gongbo2007@hotmail.com

E-mail: zmy_daisy@163.com

*Contributed equally

Key words: familial adenomatous polyposis, Adenomatous polyposis coli, next-generation sequencing, heterozygous mutation
$A P C$ gene in the Chinese population, but it also increases the understanding of the phenotypic and genotypic correlations of FAP, and may potentially lead to improved genetic counseling and specific treatment for families with FAP in the future.

\section{Introduction}

Familial adenomatous polyposis (FAP) is an autosomal dominant inherited disorder with an incidence of approximately $3-10 / 100,000$ (1). FAP is characterized by the presence of hundreds to thousands of adenomatous polyps in the colon and rectum, and is a disease predisposing individuals to colorectal cancer (CRC) (2). Affected FAP subjects without early diagnosis and treatment subsequently develop CRC in the late childhood to the sixties, with a mean age at diagnosis of 40 years old (3). CRC is the third most common malignant disease in both males and females in Asia, and is the second leading cause of cancer death in the past ten years (4). Patients who suffer from FAP also have increased risk of extra-colonic manifestations, including duodenal polyposis, sebaceous cysts, congenital hypertrophy of the retinal pigment epithelium (CHRPE) and tumors in the upper gastrointestinal tract, thyroid gland and brain $(5,6)$.

Germline mutations in the tumor suppressor adenomatous polyposis coli gene (APC) on chromosome 5q22.2 are responsible for the most cases of FAP. The APC gene comprises of 16 exons (NM_000038.5), including1 upstream non-coding exon and 15 coding exons. The last coding exon encompasses the majority $(>75 \%)$ of the total coding region. Most of the mutations causing FAP are nonsense or frameshift mutations, and can result in premature stop codons thus produce truncated APC proteins (7). The APC protein, which comprises of 2843 amino acids, plays an important role in the $\beta$-catenin nuclear localization (8). Loss of APC function results in increased level of $\beta$-catenin and activation of growth-promoting genes 
via the increased $\beta$-catenin/Tcf- 4 transcription complexes, subsequently leading to the development of adenomatous colorectal polyps at a young age (9). Adenomatous polyposis will consequently progress to CRC if left untreated.

Genetic screening along with other preventative strategies can conspicuously improve the overall survival of FAP patients. In the present study, we screened for novel mutations that might cause FAP in a four-generation Chinese family with FAP using targeted next-generation sequencing method. A novel mutation in the exon 10 of $A P C$ (c.1317delA) was identified in all of the affected members, but not in the unaffected members or the 600 normal controls. In addition, this mutation was correlated to extra-colonic phenotypes featuring by duodenal polyposis and sebaceous cysts in this pedigree. This novel mutation can hopefully be utilized as a biomarker for molecular diagnosis and precise treatment in the foreseeable future.

\section{Materials and methods}

Subjects. The family with FAP was recruited from Hospital of University of Electronic Science and Technology of China and Sichuan Provincial People's Hospital (Fig. 1). This study was conducted in accordance to the tenets of the Declaration of Helsinki and approved by the Institutional Review Boards of Sichuan Academy of Medical Sciences and Sichuan Provincial People's Hospital. Written informed consents were obtained from the family prior to the study. 600 unrelated normal control subjects were recruited from participants who attended to the annual physical examination in Sichuan Provincial People's Hospital. They underwent comprehensive examinations and were free from any related diseases.

Clinical diagnosis. Twelve of the family members were diagnosed with FAP. Non-consanguineous marriages were found in the four-generation family and their clinical information is summarized in Table I. All the available members in this family were enrolled in our study and underwent complete clinical examination. Patient III: 7, diagnosed and treated in Sichuan Provincial People's Hospital, was the proband of this family (Fig. 1). FAP was confirmed in the family by endoscopic screening. The diagnostic criteria were as follows: (1) more than 100 colorectal polyps in total, and (2) at least 20 synchronous adenomatous polyps (Fig. 2). All patients' clinical information, family history, and the results of colonoscopic, laboratory, and pathologic examinations were collected. We obtained 5-10 $\mathrm{ml}$ of peripheral blood from as many families members as possible, with full informed consent, and reviewed pathologic slides whenever available.

DNA extraction. All genomic DNA was extracted from peripheral blood using a blood DNA extraction kit (QIAamp DNA Blood Midi kit; Qiagen GmbH, Hilden, Germany) according to the manufacturer's protocol. DNA samples were stored at $-20^{\circ} \mathrm{C}$ until used. DNA integrity was evaluated by $1 \%$ agarose gel electrophoresis and NanoDrop 2000 (Thermo Fisher Scientific, Inc., Waltham, MA, USA).

Targeted next-generation sequencing and variant detection. In accordance with the literatures searched within online databases, a total of 200 candidate genes associated with colorectal cancer and ID/DD were selected as the genes of interest. We used a custom-designed gene panel, synthesized using the Agilent Sure-Select Target Enrichment technique (Zhongguancun Huakang Gene Institute, China), to capture the coding regions from the 200 genes, including their exons and exon-intron boundaries (1.285M bp in total). The following targeted next-generation sequencing (NGS) was performed on an Illumina GAIIx platform (Illumina, Inc., San Diego, CA, USA) using paired-end sequencing of $110 \mathrm{bp}$ for two patients (III:7 and III:9). Bioinformatics analysis of the raw data included the following steps: i) image analysis using RTA software version 1.9 (real-time analysis, Illumina); ii) base calling using CASAVA software v1.8.2 (Illumina); iii) filtered out duplicate and low base quality score reads using the Genome Analysis Tool kit (GATK; Broad Institute) version 4 ; iv) aligned clean paired-end reads to the human reference genome build hg19 using BWA software (Pittsburgh Supercomputing Center, Pittsburgh, PA, U.S.A.) and v) identified insertion-deletions (indels) and single-nucleotide polymorphisms (SNPs) using the GATK and annotated using ANNOVAR (version 2017, July 16). The sequencing depth was more than $5 \mathrm{X}$ (range of 5X-185X; average of 136X), and the mean coverage was $98.56 \%$. The detected variants were annotated and filtered based on public and in-house databases: i) Variants within intergenic, intronic, and UTR regions and synonymous mutations were excluded from downstream analysis and ii) variants in dbSNP138 (http://www.ncbi.nlm. nih.gov/projects/SNP/),1000GenomesProject(ftp://ftp.1000genomes. ebi.ac.uk/vol1/ftp), YH Database (http://yh.genomics.org.cn/), and HapMap Project (ftp://ftp.ncbi.nlm.nih.gov/hapmap) were excluded. Heterozygous variations of genes with autosomal dominant heredity were regarded as likely causative variations. We performed validation and parental origin analyses for these variations by conventional Sanger sequencing, and confirmed causative mutations according to parental origin of the variations and clinical features of the patients.

Mutation validation. The heterozygous APC (NM_000038.5) mutation identified by targeted NGS was further confirmed in all the family members and 600 normal controls by direct sequencing. Primers flanking the mutation were designed based on genomic sequences of Human Genome database and synthesized by Invitrogen; Thermo Fisher Scientific, Inc.: APC-F: GGGTTATATTAGTGATCCCTGCA; APC-R: ACA CAGAGGAAGCAGCTGAT. Amplified PCR products were purified with spin columns (QIAquick; Qiagen, Inc., Valencia, CA, USA) and sequenced directly (BigDye Terminators Sequencing kit; Applied Biosystems; Thermo Fisher Scientific, Inc.) in both directions with an automated genetic analysis system (3730; ABI; Thermo Fisher Scientific, Inc.). Multiple sequence alignment of the human $A P C$ protein was performed along with other APC protein across different species, to check for the conservation of the residues. The homology modelling server SWISS-MODEL (www.swissmodel.expasy.org/) was used to predict the tertiary structure of APC protein.

Brief Literature review. A systematic literature search was conducted using the PubMed, Embase, Web of Science, and the Chinese Biomedical Database, in order to identify all 


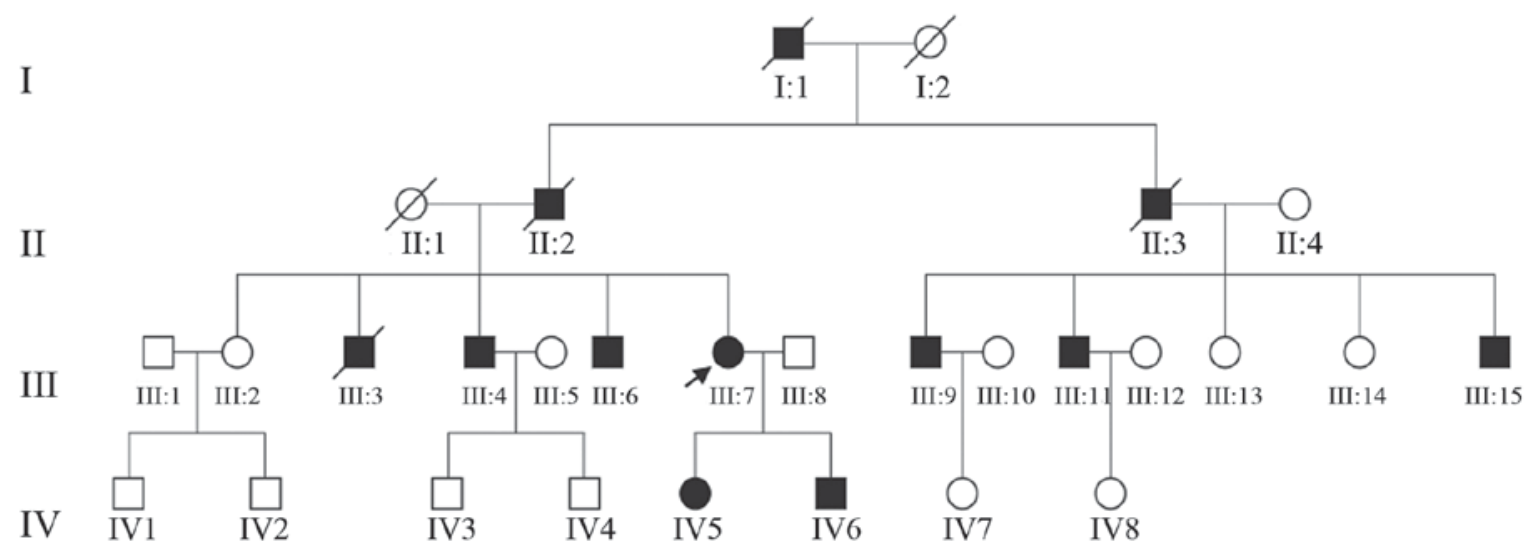

Figure 1. Pedigree of the FAP family. A four-generation family composed of 29 members from Sichuan, China, was recruited in the present study. The black arrow denotes the proband, solid symbols indicate affected individuals, and open symbols indicate unaffected individuals. In total, 12 family members were diagnosed with FAP. The disease exhibited a pattern of autosomal dominant inheritance. FAP, familial adenomatous polyposis; squares, males; circles, females; symbols with diagonal lines, deceased.

published studies on the $A P C$ mutations of Han Chinese FAP subjects from their starting date to December 31, 2017. Two observers (YL and MP) independently piled up data from all eligible publications onto paper data collection forms. Disagreements were resolved by discussion until a consensus was achieved. Otherwise, two other investigators (BG and $\mathrm{ZY}$ ) were consulted to resolve the dispute. The search strategy was based on the following search term/phase, 'Familial adenomatous polyposis or FAP', 'adenomatous polyposis coli gene or $A P C$ ' and 'Chinese'. The eligible articles were considered if they: i) evaluated germline mutations of $A P C$ gene in Chinese FAP families and ii) were original research articles, not reviews or comments. The articles selected were limited to studies in humans and in Han Chinese, but without restriction on sample size or time period. Excluded were abstracts from conferences, full texts without raw data available for retrieval, republished data, duplicate studies, association studies and reviews. A total of 79 Han Chinese families from 20 independent studies (including the current study) were included (some of the studies reported more than one family). All the mutations in the $A P C$ gene were coordinated using the same transcript reference NM_000038.5.

\section{Results}

Clinical findings. A four-generation family composed of 29 members from Sichuan Province of China was recruited in this study. Among them, I1, I2, II1, II2, and II3 were deceased before this study, their information were collected from their medical records. III3 was deceased after he participated in this study and donated his blood sample. In total, 12 family members were diagnosed with FAP according to their clinical features, family history, medical history, colonoscopy, and pathology examinations. The disease exhibited a pattern of autosomal dominant inheritance, as indicated by the familial pedigree (Fig. 1). Detailed clinical information was presented in Table I.

Extracolonic manifestations featuring by duodenal polyposis and sebaceous cysts were observed in all the available patients of this family, indicating a correlation with this novel mutation. The proband (III:7) had obvious duodenal polyposis, and was diagnosed with $\mathrm{CRC}$ at the age of 42 year old; while his cousin (III:15) had an onset of CRC at 43 year old. Two grape beaded polyps and multiple small polyps were present in the transverse colon and descending colon of the proband (Fig. 2). The mean age of diagnosis of polyposis was 30.5 years old among the patients in this family. The daughter (IV:5) and son (IV:6) of the proband had an aggressive phenotype with early onset of the polyposis at younger age of 16 and 21 years old, respectively.

Targeted next-generation sequencing and mutation validation. To ensure complete sequencing coverage of all coding regions in $A P C$, the quality and reliability of NGS data were evaluated based on the percentage of readable bases and the coverage depth in the targeted region. In the $A P C$ gene, the coverage depth was up to $200 x$, with $100 \%$ of bases being readable in coding regions. This suggests high capacity for variant identification in most of the exons. Additionally, the mean depth was close to the median depth in each exon, indicating a good randomicity. On average, 295 variations within the 200 genes were found in the two patients (III:7 and III:9). Under the autosomal dominant model, 8 novel variations were filtered out. Because previously unclassified variations and pathogenic mutations detected by NGS were considered as causative candidate, the filtered data was narrowed down to a pathogenic heterozygous variant (c.1317delA in the $A P C$ gene). This variant was further validated using Sanger sequencing on other family members and 600 normal controls. Finally, we confirmed the novel heterozygous deletion mutation c.1317delA (p.Ala440LeufsTer14; Fig. 3A) in exon 10 of $A P C$ in the all of the affected members, and this mutation was absent in the unaffected member and the other 600 normal controls. Genotypes and phenotypes for the patients with $A P C$ mutations were described in Table I. Therefore, this mutation was co-segregated with the phenotype in this family. Comparative amino acid sequence alignment of other APC protein across different species revealed that this novel mutation occurred at highly conserved positions (Fig. 3B). This mutation is a 1-bp deletion of $\mathrm{A}$ at exon 10 (c.1317delA), leading to a subsequent reading frame-shift and producing a premature termination codon located at the 454th amino 
Table I. Clinical information of the participants in the family.

\begin{tabular}{|c|c|c|c|c|c|c|c|c|}
\hline $\begin{array}{l}\text { Family } \\
\text { ID }\end{array}$ & Gender & $\begin{array}{c}\text { Age } \\
\text { (years) }\end{array}$ & Diagnosis & $\begin{array}{c}\text { Age at } \\
\text { diagnosis }\end{array}$ & Mutation & $\begin{array}{l}\text { Polyp } \\
\text { count }\end{array}$ & $\begin{array}{c}\text { CRC } \\
\text { (age of onset) }\end{array}$ & $\begin{array}{c}\text { Extra-colonic } \\
\text { features }\end{array}$ \\
\hline $\mathrm{I}: 1^{\mathrm{a}}$ & M & Deceased & Patient & Unspecified & Unspecified & Unspecified & Unspecified & Unspecified \\
\hline $\mathrm{I}: 2^{\mathrm{a}}$ & $\mathrm{F}$ & Deceased & Normal & 1 & / & I & I & 1 \\
\hline II: $1^{\mathrm{a}}$ & $\mathrm{F}$ & Deceased & Normal & I & I & l & I & I \\
\hline $\mathrm{II}: 2^{\mathrm{a}}$ & M & Deceased & Patient & Unspecified & Unspecified & Unspecified & Unspecified & Unspecified \\
\hline II: $3^{\mathrm{a}}$ & M & Deceased & Patient & Unspecified & Unspecified & Unspecified & Unspecified & Unspecified \\
\hline II:4 & $\mathrm{F}$ & 75 & Normal & I & I & I & 1 & 1 \\
\hline III:1 & $\mathrm{M}$ & 64 & Normal & I & / & / & l & / \\
\hline III:2 & $\mathrm{F}$ & 62 & Normal & / & l & / & / & l \\
\hline III: $3^{\mathrm{b}}$ & M & Deceased & Patient & 34 & c.1317delA & $>100$ & Unspecified & None \\
\hline III:4 & M & 47 & Patient & 37 & c.1317delA & $>100$ & None & None \\
\hline III:5 & $\mathrm{F}$ & 46 & Normal & 1 & / & / & 1 & I \\
\hline III:6 & M & 45 & Patient & 38 & c.1317delA & $>100$ & None & None \\
\hline III:7 & $\mathrm{F}$ & 43 & Patient & 36 & c.1317delA & $>100$ & 42 & None \\
\hline III: 8 & M & 45 & Normal & / & I & 1 & 1 & I \\
\hline III:9 & M & 53 & Patient & 44 & c.1317delA & $>100$ & None & None \\
\hline III:10 & $\mathrm{F}$ & 50 & Normal & 1 & I & 1 & l & 1 \\
\hline III:11 & M & 50 & Patient & 49 & c.1317delA & $>100$ & None & None \\
\hline III:12 & $\mathrm{F}$ & 48 & Normal & / & I & I & / & / \\
\hline III:13 & $\mathrm{F}$ & 48 & Normal & l & / & / & l & / \\
\hline III:14 & $\mathrm{F}$ & 47 & Normal & I & l & l & I & l \\
\hline III:15 & M & 43 & Patient & 40 & c.1317delA & $>100$ & 43 & None \\
\hline IV:1 & M & 39 & Normal & / & / & / & / & 1 \\
\hline IV:2 & M & 37 & Normal & I & I & / & I & l \\
\hline IV:3 & M & 24 & Normal & l & l & / & l & / \\
\hline IV:4 & M & 12 & Normal & I & / & l & l & / \\
\hline IV:5 & $\mathrm{F}$ & 24 & Patient & 21 & c.1317delA & $>100$ & None & None \\
\hline IV:6 & M & 19 & Patient & 16 & c.1317delA & $>100$ & None & None \\
\hline IV:7 & $\mathrm{F}$ & 20 & Normal & / & / & / & / & / \\
\hline IV:8 & $\mathrm{F}$ & 22 & Normal & I & I & I & I & I \\
\hline
\end{tabular}

${ }^{a}$ I1, I2, III, II2, II3 were deceased prior to the present study, their information was collected from their medical records. bIII3 was deceased following participation in the present study and donated his blood sample. M, male; F, female; /, not applicable; CRC, colorectal cancer.

acid downstream (p.Ala440LeufsTer14). This premature termination consequently resulted in a truncation of 2,389 amino acids downstream of APC protein (Fig. 4). To further study the pathogenicity of this mutation, protein tertiary structures of wild type and p.Ala440LeufsTer14 mutation of APC were predicted using the SWISS-MODEL. According to the tetramer, the mutated APC protein lost C-terminal helices (2,389 amino acids downstream), which was an important region to bind with $\beta$-catenin, microtubule and other elements (Fig. 4).

Brief literature review of the APC mutations in Han Chinese. All the mutations that were hitherto reported were listed in Table II. We have retrieved 20 individual studies (including the current study). In total, there were 79 Han Chinese FAP families included, and 54 different $A P C$ mutations reported. Among the mutations, 59.3\% (32/54) were frameshift mutations, $24.1 \%(13 / 54)$ were nonsense mutations, $3.7 \%(2 / 54)$ were missense mutations, $7.4 \%$ (4/54) were large deletions and $5.5 \%(3 / 54)$ were intronic variants.

There were three main hotspot mutation sites in the $A P C$ gene: i) c.3180-c.3187/codon 1061-1062, which was in the $\beta$-catenin binding domain of $A P C$, accounted for $12.7 \%(10 / 79)$ of the occurrence; ii) c.3921-c.3931/codon 1307-1309, which was in the mutation cluster region (MCR), accounted for $25.3 \%$ (20/79) of the occurrence; and iii) c.5432C >T (p.Ser1811Leu), which was in the $\beta$-catenin binding and down-regulation domain, accounted for $3.8 \%$ (3/79) of the occurrence. In total, these hotspot mutations accounted for $41.8 \%$ of the overall occurrence.

\section{Discussion}

Familial adenomatous polyposis (FAP) refers to an autosomal dominant inherited condition, which is characterized by the early onset of numerous adenomatous colorectal polyps. 

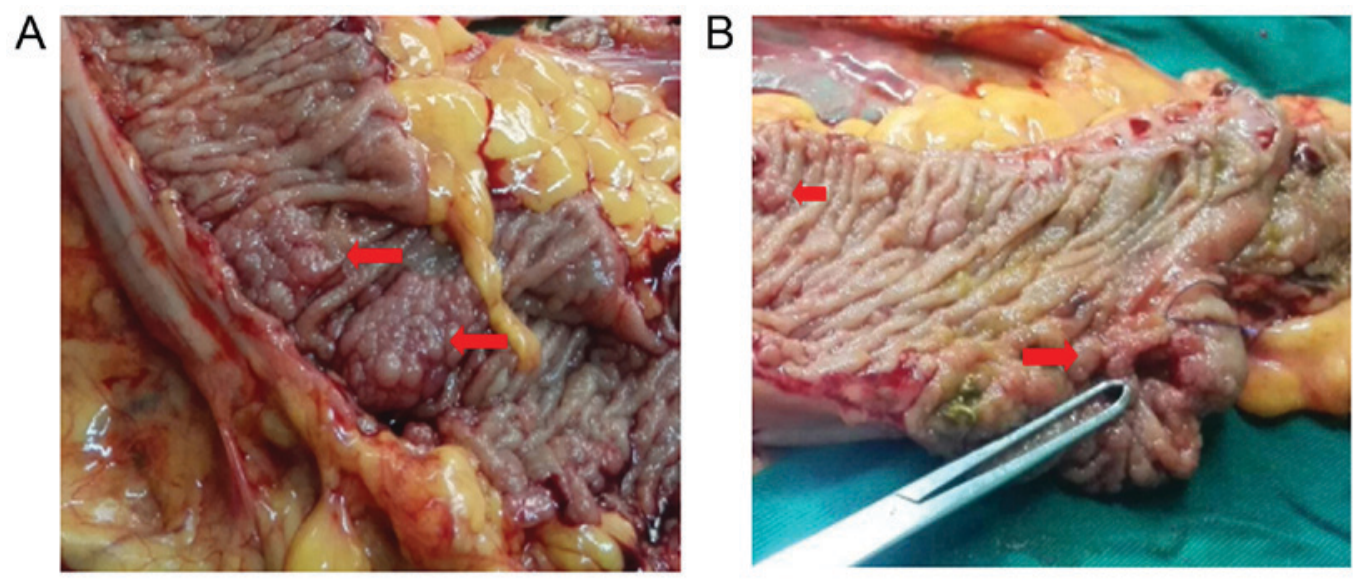

Figure 2. Clinical characterization of the proband of this pedigree. (A) Two grape beaded polyps (red arrows) and some small polyps in the transverse colon. (B) A grape beaded polyp and some small polyps (red arrows) in the transverse colon.

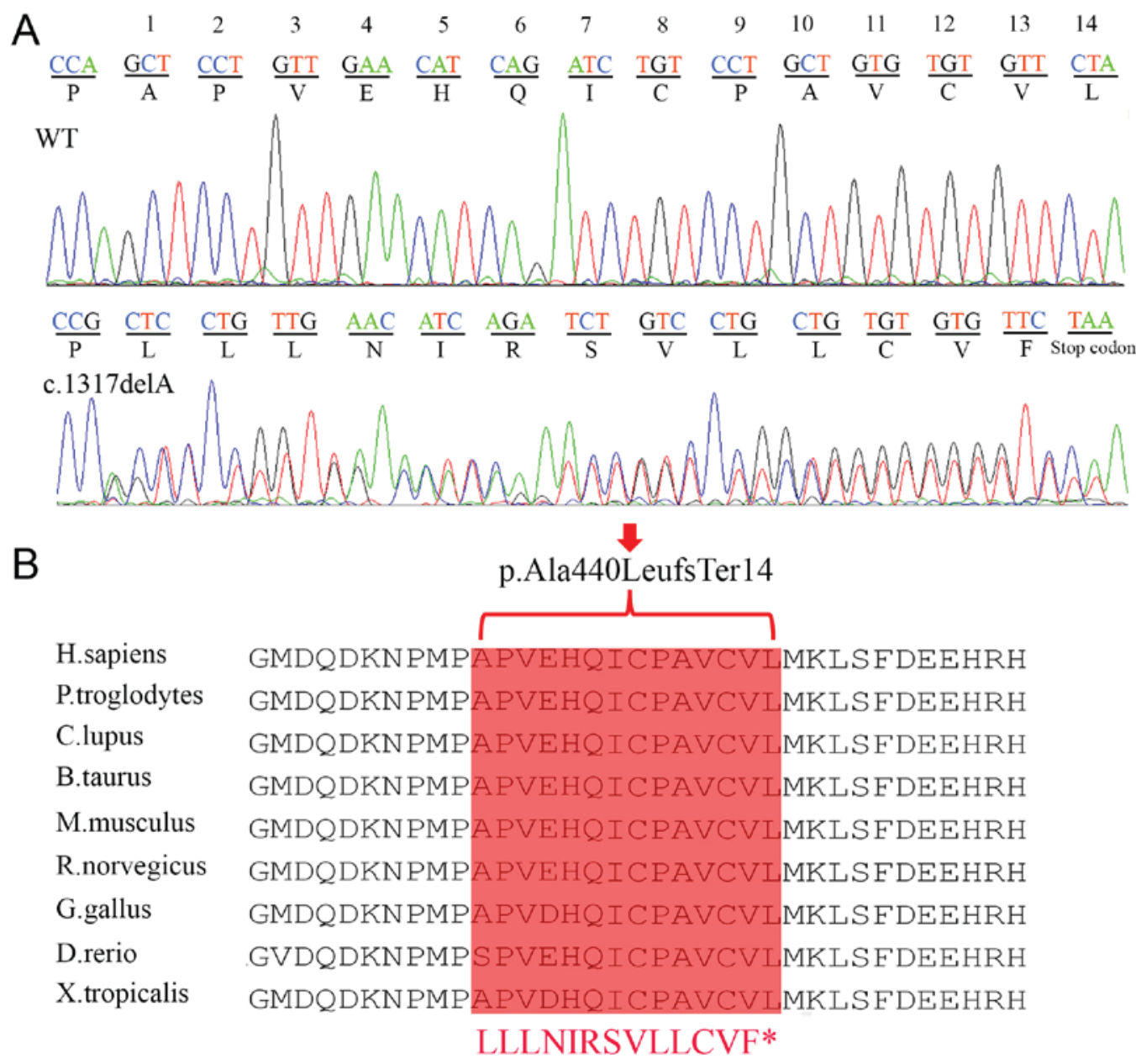

Figure 3. Novel mutation in the APC gene in this pedigree. (A) The novel heterozygous mutation c.1317delA (p.Ala440LysfsTer14) was validated using Sanger sequencing. (B) Multiple sequence alignment of APC proteins from various species. Red shades showed the premature termination of amino acids, which was resulted from the deletion. This region is highly conserved acrossdifferent species. APC, Adenomatous polyposis coli.

Approximately $50 \%$ of FAP patients develop adenomas at the age of 15 , and it increases to $95 \%$ by the age of 35 years old (10). In many FAP patients, extra colonic manifestations are observed, including gastric and duodenal polyps, congenital hypertrophy of the retinal pigment epithelium (CHRPE), sebaceous cysts, and tumors in the upper gastrointestinal tract, thyroid gland and brain $(5,6)$. In this pedigree, a novel frameshift mutation c.1317delA (p.Ala440LeufsTer14) in the $A P C$ gene was identified. The proband has the heterozygous mutation, so theoretically he inherited the mutant strand from his father (who was also a patient) and the wild-type strand from his mother (who was healthy). But unfortunately we are 


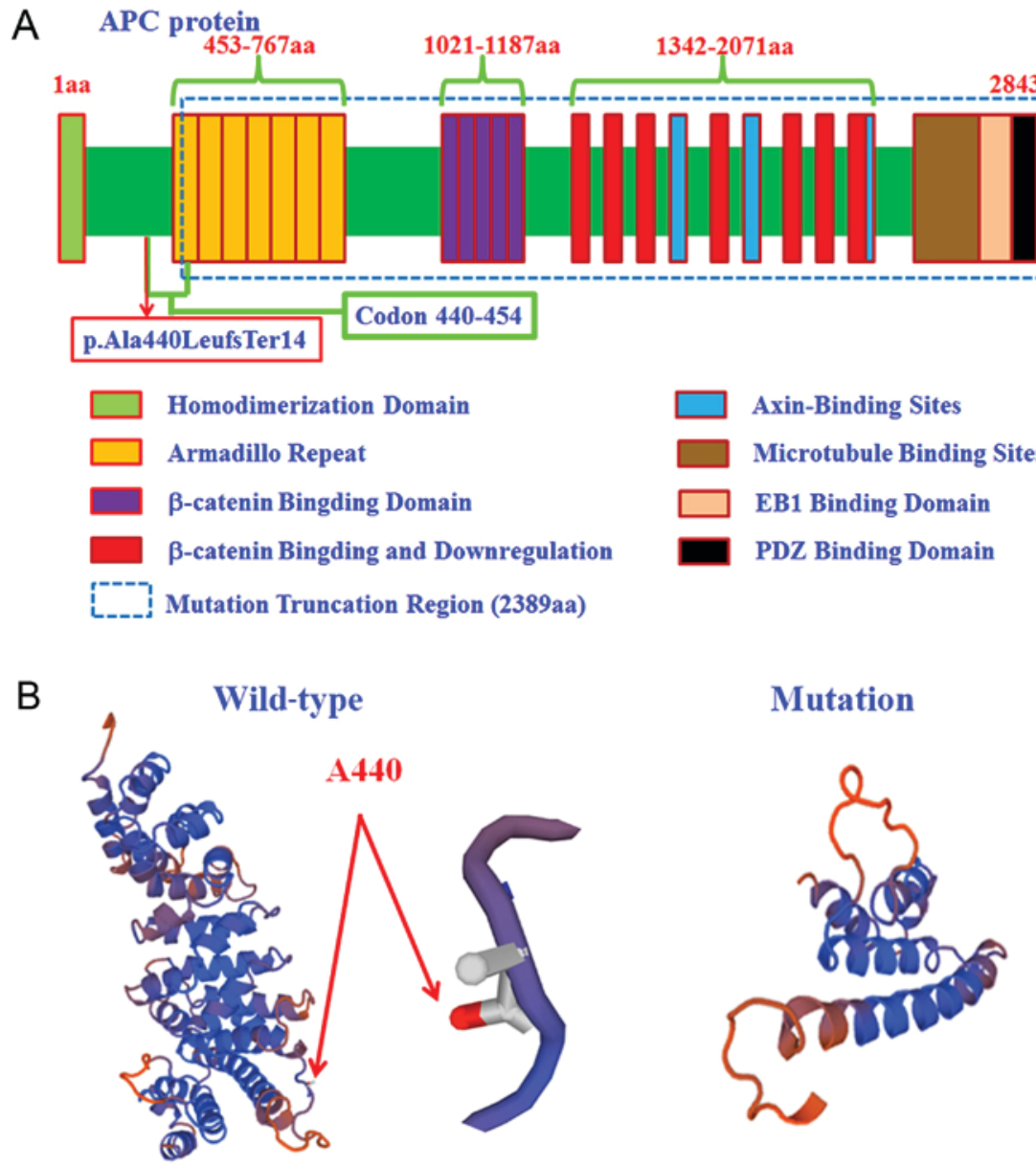

Figure 4. Structure modeling of the functional domains of APC protein. (A) Structure of the APC protein, the red box denotes the site of the novel mutation, the green box shows the premature termination codon, the box with a dashed blue line indicates the truncated region of this protein, and the colored squares indicate the sub-domains in the APC protein. (B) Structure modeling of wild type and p.Ala440LeufsTer14 mutation of APC with SWISS-MODEL. Compared with wild type, the mutant leads to premature termination and potential loss of ability to bind with $\beta$-catenin. A440, Ala440; aa, amino acids; APC, Adenomatous polyposis coli.

not able to validate the phenotypes of the proband's father and mother, because both of them passed away. However, we noticed that the novel mutation was correlated to extra colonic manifestations featuring by duodenal polyposis and sebaceous cysts. In addition, FAP patients also have a high lifetime risk of colorectal cancer (CRC), which is one of the most common forms of cancer in the world $(10,11)$. In this family, patient III:7 (proband) and III:15 have been already diagnosed with CRC at the age of 42 and 43 respectively. Considering FAP-related CRC takes many years to develop, and the overall mean age of onset for CRC is 40 years old (3), unfortunately it's possible that other patients in this family may still have a high risk of CRC as time goes by. As a result, early diagnosis and routine medical monitoring are remarkably crucial for the FAP patients.

Genetic diagnosis mainly focuses on the $A P C$ gene in chromosome $5 \mathrm{q} 22.2$, as mutations in this gene almost account for all cases of FAP (12). The APC gene encodes a tumor suppressor protein, which is responsible for ubiquitination and degradation of $\beta$-catenin and functions as an antagonist of the Wnt signaling pathway $(13,14)$. $\beta$-catenin is an important factor in cell cycle entry, proliferation, differentiation, migration, apoptosis, and progression. Abnormal accumulation of $\beta$-catenin and aberrant activation of $\mathrm{Wnt} / \beta$-catenin pathway leads to progression of several major human cancers $(15,16)$. In addition, APC also involves in other processes including cell migration and adhesion, transcriptional activation, microtubule stabilization and apoptosis. Inactivation of APC can lead to defective chromosome segregation and aberrant mitosis (17-19). In the present study, we detected a novel frame shift mutation c.1317delA (p.Ala440LeufsTer14) in the exon 10 of the $A P C$ gene in a large Chinese FAP pedigree. This mutation could result in a truncation of 2,389 amino acids downstream of APC protein. Protein tertiary structure analysis revealed that the mutated APC protein lost C-terminal helices, which was an important region to bind with $\beta$-catenin, microtubule and other elements.

Hitherto, more than 4,600 APC mutations have been reported according to Leiden Open Variation Database (LOVD; databases.lovd.nl/shared/genes/APC). However, these mutations are related to numerous diseases, mainly gastric cancer. And they are not confined to a certain population. Given that FAP-related CRC only accounts for approximately $1 \%$ of overall CRC (2) and ethnical difference might exist among different populations, we conducted a brief literature review and focused on the $A P C$ mutation spectrum only in the Han Chinese FAP population. According to our findings, among the 4,600 reported mutations, only 54 were 
Table II. Reported APC mutations causing familial adenomatous polyposis in the Han Chinese population.

\begin{tabular}{|c|c|c|c|c|c|}
\hline Author, year & Mutation in $A P C$ gene (Han Chinese) & Codon & Mutation types & Occurrence & (Refs.) \\
\hline Liu et al, 2005 & c.366delG(p.Phe123LeufsX2) & 123 & Frameshift & 1 & $(20)$ \\
\hline Liu et al, 2005 & c.387insT(p.Glu129AspfsX10) & 129 & Frameshift & 1 & $(20)$ \\
\hline Liu et al, 2005 & c.541insA(p.Gln181ThrfsX12) & 181 & Frameshift & 1 & $(20)$ \\
\hline Liu et al, 2005 & c. $637 \mathrm{C}>\mathrm{T}(\mathrm{p} . \operatorname{Arg} 213 \mathrm{X})$ & 213 & Nonsense & 1 & $(20)$ \\
\hline Pang et al, 2001 & c.646C>T (p.Arg216X) & 216 & Nonsense & 1 & $(21)$ \\
\hline Cai et al, 2008 & c. $694 \mathrm{C}>\mathrm{T}(\mathrm{p} . \operatorname{Arg} 232 \mathrm{X})$ & 232 & Nonsense & 1 & $(22)$ \\
\hline Cai et al, 2008 & c.763_764insA(p.His255GlnfsX2) & 255 & Frameshift & 1 & $(22)$ \\
\hline Zhang et al, 2016 & c.794_795insG (p.Val266SerfsX11) & 266 & Frameshift & 1 & $(23)$ \\
\hline $\begin{array}{l}\text { Liu et al, 2005; } \\
\text { Pang et al, } 2001\end{array}$ & c.847C>T(p.Arg283X) & 283 & Nonsense & 2 & $(20,21)$ \\
\hline Liu et al, 2005 & c. $1213 \mathrm{C}>\mathrm{T}(\mathrm{p} . \operatorname{Arg} 405 \mathrm{X})$ & 405 & Nonsense & 1 & $(20)$ \\
\hline Pang et al, 2018 & c.1317delA (p.Ala440LeufsX14) & 440 & Frameshift & Novel & $\begin{array}{l}\text { The present } \\
\text { study }\end{array}$ \\
\hline Sheng et al, 2010 & c.1327G > T (p.Glu443X) & 443 & Nonsense & 1 & $(24)$ \\
\hline Liu et al, 2005 & c. $1495 \mathrm{C}>\mathrm{T}(\mathrm{p} . \operatorname{Arg} 499 \mathrm{X})$ & 499 & Nonsense & 1 & $(20)$ \\
\hline Jang et al, 2010 & c.1548G >C (p.Lys516Asn) & 516 & Missense & 1 & $(25)$ \\
\hline Jang et al, 2010 & c.1766T>A (p.Leu589X) & 589 & Nonsense & 1 & $(25)$ \\
\hline Song et al, 2013 & $\begin{array}{l}\text { c.1828_1829insG } \\
\text { (p.Asp610GlyfsX23) }\end{array}$ & 610 & Frameshift & 1 & $(26)$ \\
\hline Chen et al, 2011 & c.1999 C>T (p. Gln667X) & 667 & Nonsense & 1 & $(27)$ \\
\hline Jang et al, 2010 & $\begin{array}{l}\text { c.2016_2047del } \\
\text { (p.Ser673LeufsX10) }\end{array}$ & 673 & Frameshift & 1 & $(25)$ \\
\hline Cai et al, 2008 & $\begin{array}{l}\text { c.2031_2034delCAGT } \\
\text { (p.Ser678MetfsX39) }\end{array}$ & 678 & Frameshift & 1 & $(22)$ \\
\hline Zhang et al, 2016 & $\begin{array}{l}\text { c.2142_2143insG } \\
\text { (p.His715AlafsX19) }\end{array}$ & 715 & Frameshift & 1 & $(23)$ \\
\hline Cai et al, 2008 & c. $2240 \mathrm{C}>\mathrm{G}(\mathrm{p} . \operatorname{Ser} 747 \mathrm{X})$ & 747 & Nonsense & 1 & $(22)$ \\
\hline Sheng et al, 2010 & $\begin{array}{l}\text { c.2336_2337insT } \\
\text { (p.Leu779PhefX9) }\end{array}$ & 779 & Frameshift & 1 & $(24)$ \\
\hline Jang et al, 2010 & c. $2510 C>G(p . S e r 837 X)$ & 837 & Nonsense & 1 & $(25)$ \\
\hline Liu et al, 2005 & $\begin{array}{l}\text { c.2547_2550delTAGA } \\
\text { (p.Asp849GlufsX11) }\end{array}$ & 849 & Frameshift & 1 & $(20)$ \\
\hline Li et al, 2017 & c. $2971 \mathrm{G}>$ T (p.Glu991X) & 991 & Nonsense & 1 & $(28)$ \\
\hline Pang et al, 2001 & $\begin{array}{l}\text { c.3067_3068insA } \\
\text { (p.Thr1023AsnfsX6) }\end{array}$ & 1,023 & Frameshift & 1 & $(21)$ \\
\hline Cai et al, 2008 & $\begin{array}{l}\text { c.3182_3183delAA } \\
\left(\text { p.Lys1061ThrfsX3) }{ }^{\mathrm{a}}\right.\end{array}$ & 1,061 & Frameshift & 1 & $(22)$ \\
\hline $\begin{array}{l}\text { Liu et al, 2005; } \\
\text { Pang et al, 2001; } \\
\text { Cai et al, 2008; } \\
\text { Sheng et al, } 2010\end{array}$ & $\begin{array}{l}\text { c.3183_3187delACAAA } \\
(\text { p.Lys1061LysfsX2)a }\end{array}$ & 1,061 & Frameshift & 5 & $(20-24)$ \\
\hline $\begin{array}{l}\text { Wang et al, 2008; } \\
\text { Chen et al, 2015; } \\
\text { Chen et al, } 2015\end{array}$ & $\begin{array}{l}\text { c3184_3187delCAAA } \\
\text { (p.Gln1061AspfsX59) }\end{array}$ & 1,061 & Frameshift & 3 & $(29-31)$ \\
\hline Jang et al, 2010 & $\begin{array}{l}\text { c.3180_3184del } \\
(\text { p.Gln1062X) }\end{array}$ & 1,062 & Nonsense & 1 & 25 \\
\hline
\end{tabular}


Table II. Continued.

\begin{tabular}{|c|c|c|c|c|c|}
\hline Author, year & Mutation in APC gene (Han Chinese) & Codon & Mutation types & Occurrence & (Refs.) \\
\hline $\begin{array}{l}\text { Cai et al, 2008; } \\
\text { Sheng et al, } 2010\end{array}$ & $\begin{array}{l}\text { c.3202_3205delTCAA } \\
\text { (p.Ser1068GlyfsX57) }\end{array}$ & 1,068 & Frameshift & 3 & $(22,24)$ \\
\hline Zhang et al, 2017 & c.3418delC ( p.Pro1140Leufx25) & 1,140 & Frameshift & 1 & $(32)$ \\
\hline Cai et al, 2008 & c.3576delA(p.Lys1192AsnfsX73) & 1,192 & Frameshift & 1 & $(22)$ \\
\hline Zhu et al, 2012 & c. $3587 \mathrm{C}>\mathrm{A}(\mathrm{p} . \operatorname{Ser} 1196 \mathrm{X})$ & 1,196 & Frameshift & 1 & $(33)$ \\
\hline Liu et al, 2005 & c.3667delC (p.Ser1223LeufsX42) & 1,223 & Frameshift & 1 & $(20)$ \\
\hline Chen et al, 2015 & $\begin{array}{l}\text { c.3921_3924delAAAA } \\
(\text { p.Ile1307MetfsX13) }\end{array}$ & 1,307 & Frameshift & 1 & $(31)$ \\
\hline Sheng et al, 2010 & $\begin{array}{l}\text { c.3923_3929delAAGAAAA } \\
\left(\text { p.Lys1308ArgfsX11) }{ }^{\mathrm{a}}\right.\end{array}$ & 1,308 & Frameshift & 1 & (24) \\
\hline Chen et al, 2015 & c.3926_3929delAAAA ${ }^{\mathrm{a}}$ & 1,309 & Frameshift & 1 & $(30)$ \\
\hline Liao et al, 2014 & $\begin{array}{l}\text { c.3922_3925 del AAAG } \\
\text { (p. Glu1309Argfs X11) }\end{array}$ & 1,309 & Frameshift & 1 & $(34)$ \\
\hline $\begin{array}{l}\text { Wang et al, 2008; } \\
\text { Chen et al, 2015; } \\
\text { Chen et al, 2015; } \\
\text { Liao et al, } 2014\end{array}$ & $\begin{array}{l}\text { c.3926_3930delAAAAG } \\
\text { (p.Glu1309AspfsX4) }\end{array}$ & 1,309 & Frameshift & 6 & $(29-31,34)$ \\
\hline $\begin{array}{l}\text { Liu et al, 2005; } \\
\text { Cai et al, 2008; } \\
\text { Sheng et al, 2010; } \\
\text { Zhang et al, 2016; } \\
\text { Pan et al, 2014; } \\
\text { Gan et al, } 1994\end{array}$ & $\begin{array}{l}\text { c.3927_3931delAAAGA } \\
\text { (p.Glu1309AspfsX4) }\end{array}$ & 1,309 & Frameshift & 10 & $\begin{array}{c}(20,22,24, \\
35-37)\end{array}$ \\
\hline Chen et al, 2015 & $\begin{array}{l}\text { c4127_4126delAT } \\
\text { (p.Tyr1376LysfsX9) }\end{array}$ & 1,376 & Frameshift & 1 & $(31)$ \\
\hline Sheng et al, 2010 & $\begin{array}{l}\text { c.4179_4180GAdelinsT } \\
\text { (p.Asp1394LeufsX21) }\end{array}$ & 1,394 & Frameshift & 1 & $(24)$ \\
\hline Cai et al, 2008 & $\begin{array}{l}\text { c.4209delC } \\
\text { (p.Ser1404ProfsX11) }\end{array}$ & 1,404 & Frameshift & 1 & $(22)$ \\
\hline Liu et al, 2005 & $\begin{array}{l}\text { c.4391_4394delAGAG } \\
\text { (p.Glu1464ValfsX8) }\end{array}$ & 1,464 & Frameshift & 1 & $(20)$ \\
\hline $\begin{array}{l}\text { Wang et al, 2008; } \\
\text { Chen et al, 2015; } \\
\text { Chen et al, } 2015\end{array}$ & c. $5432 \mathrm{C}>\mathrm{T}(\mathrm{p} . \text { Ser1811Leu })^{\mathrm{a}}$ & 1,811 & Missense & 3 & $(29-31)$ \\
\hline Liu et al, 2005 & $\begin{array}{l}\text { c.5947_5950delGAAA } \\
\text { (p.Glu1983MetfsX60) }\end{array}$ & 1,983 & Frameshift & 1 & $(20)$ \\
\hline Jiang et al, 2015 & c. $1936-2148$ del & l & Large deletion & 1 & (3) \\
\hline Sheng et al, 2010 & $\begin{array}{l}\text { 10A (Alternative exon) } \\
\text { and Exon } 11\end{array}$ & l & Large deletion & 1 & (24) \\
\hline Sheng et al, 2010 & Exon15 start & l & Large deletion & 1 & $(24)$ \\
\hline Zhang et al, 2016 & c.423_8532del & l & Large deletion & 1 & $(38)$ \\
\hline Sheng et al, 2010 & c. $.532-2 \mathrm{~A}>\mathrm{T}$ & l & Intronic & 1 & $(24)$ \\
\hline Sheng et al, 2010 & c. $657+1 \mathrm{G}>\mathrm{A}$ & I & Intronic & 1 & $(24)$ \\
\hline Zhang et al, 2016 & c. $1312+1 G>A$ & l & Intronic & 1 & $(23)$ \\
\hline
\end{tabular}

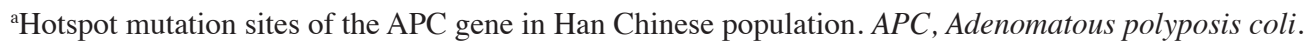


related to Han Chinese FAP. Truncating mutations, including frameshift mutations (59.3\%), nonsense mutations (24.1\%) and large deletions (7.4\%), account for an overall percentage of $90.8 \%$. While non-truncating mutations, including missense mutations (3.7\%) and intronic mutations (5.5\%), account for the rest $9.2 \%$. These findings support that direct sequencing of $A P C$ gene could still be the gold standard and the most accurate method in genetic diagnosis of FAP. In fact, there are several other alternative methods for gene testing, such as protein truncation test (PTT), multiplex ligation-dependent probe amplification (MLPA), southern blot, and real-time quantitative PCR (RT-PCR). But the major disadvantage of these methods is the inability to detect the non-truncating mutations, which account for $9.2 \%$ in the Han Chinese population. As a result, we still recommend that FAP testing should be performed using direct sequencing of the whole $A P C$ gene to detect the small-scale mutations along with MLPA to identify large insertion and deletions. If no mutation is detected, then testing for large gene rearrangements should be conducted.

To sum up, we reported that a novel heterozygous frame shift mutation c.1317delA (p.Ala440LeufsTer14) in the $A P C$ gene was responsible for FAP in a large Chinese pedigree. And we revealed a genotype-phenotype correlation between this novel mutation and extra colonic manifestations featuring by duodenal polyposis and sebaceous cysts. In the meantime, we conducted a literature review, piling up all the reported $A P C$ mutations causing FAP in the Han Chinese population. In summary, these data enhance understanding of the mutation spectrum of the $A P C$ gene causing FAP in Han Chinese and inform the development of effective guidelines in its genetic diagnosis and clinical management.

\section{Acknowledgements}

Not applicable.

\section{Funding}

The present study was supported by grants from the Natural Science Foundation of China (grant nos.81371048 and 81670853), The Department of Science and Technology of Sichuan Province, China (grant no. 2015HH0031) and The Department of Sichuan Provincial Health (grant no. 130163).

\section{Availability of data and materials}

The datasets used and analyzed during the current study are available from the corresponding authors on reasonable request.

\section{Authors' contributions}

BG and ZY designed the study. MP, XiH, XuH, NH, PL, LL, $\mathrm{JF}$ and $\mathrm{KW}$ recruited the participants and collected the clinical information. ZY and JY performed the sequencing analysis. YL and MP conducted the literature review and analyzed the data. ZY wrote the initial draft, and BG corrected the English spelling and grammar. All authors critically revised, reviewed and approved the publication of this manuscript.

\section{Ethics approval and consent to participate}

The present study was approved by the Institutional Review Boards of Sichuan Academy of Medical Sciences and Sichuan Provincial People's Hospital (Sichuan, China). Written informed consents were obtained from the family prior to the study.

\section{Consent for publication}

Written informed consents were obtained from the family prior to the study.

\section{Competing interests}

The authors declare that they have no competing interests.

\section{References}

1. Half E, Bercovich D and Rozen P: Familial adenomatous polyposis. Orphanet J Rare Dis 4: 22, 2009.

2. Galiatsatos $P$ and Foulkes WD: Familial adenomatous polyposis. Am J Gastroenterol 101: 385-398, 2006.

3. Jiang SS, Li JJ, Li Y, He LJ, Wang QJ, Weng DS, Pan K, Liu Q, Zhao JJ, Pan QZ, et al: A novel pathogenic germline mutation in the adenomatous polyposis coli gene in a Chinese family with familial adenomatous coli. Oncotarget 6: 27267-27274, 2015.

4. Sung JJ, Lau JY, Goh KL and Leung WK; Asia Pacific Working Group on Colorectal Cancer: Increasing incidence of colorectal cancer in Asia: Implications for screening. Lancet Oncol 6: 871-876, 2005.

5. Jang YH, Lim SB, Kim MJ, Chung HJ, Yoo HW, Byeon JS, Myung SJ, Lee W, Chun S and Min WK: Three novel mutations of the APC gene in Korean patients with familial adenomatous polyposis. Cancer Genet Cytogenet 200: 34-39, 2010.

6. Burt RW, DiSario JA and Cannon-Albright L: Genetics of colon cancer: Impact of inheritance on colon cancer risk. Annu Rev Med 46: 371-379, 1995.

7. Stenson PD, Ball EV, Mort M, Phillips AD, Shiel JA, Thomas NS, Abeysinghe S, Krawczak M and Cooper DN: Human gene mutation database (HGMD): 2003 update. Hum Mutat 21: 577-581, 2003.

8. Fearnhead NS, Britton MP and Bodmer WF: The ABC of APC. Hum Mol Genet 10: 721-733, 2001.

9. Korinek V, Barker N, Morin PJ, van Wichen D, de Weger R, Kinzler KW, Vogelstein B and Clevers H: Constitutive transcriptional activation by a beta-catenin-Tcf complex in APC-/- colon carcinoma. Science 275: 1784-1787, 1997.

10. Leoz ML, Carballal S, Moreira L, Ocaña T and Balaguer F: The genetic basis of familial adenomatous polyposis and its implications for clinical practice and risk management. Appl Clin Genet 8: 95-107, 2015.

11. Ibrahim A, Barnes DR, Dunlop J, Barrowdale D, Antoniou AC and Berg JN: Attenuated familial adenomatous polyposis manifests as autosomal dominant late-onset colorectal cancer. Eur J Hum Genet 22: 1330-1333, 2014.

12. Aihara H,Kumar N and Thompson CC: Diagnosis, surveillance, and treatment strategies for familial adenomatous polyposis: Rationale and update. Eur J Gastroenterol Hepatol 26: 255-262, 2014.

13. Van de Wetering M, Sancho E, Verweij C, de Lau W, Oving I, Hurlstone A, van der Horn K, Batlle E, Coudreuse D, Haramis AP, et al: The beta-catenin/TCF-4 complex imposes a crypt progenitor phenotype on colorectal cancer cells. Cell 111: 241-250, 2002.

14. Sieber OM, Tomlinson IP and Lamlum H: The adenomatous polyposis coli (APC) tumour suppressor-genetics, function and disease. Mol MedToday 6: 462-469, 2000.

15. Moon RT, Kohn AD, De Ferrari GV and Kaykas A: WNT and beta-catenin signalling: Diseases and therapies. Nat Rev Genet 5: 691-701, 2004.

16. Tang Y, Simoneau AR, Liao WX, Yi G, Hope C, Liu F, Li S, Xie J, Holcombe RF, Jurnak FA, et al: WIF1, a Wnt pathway inhibitor, regulates SKP2 and c-myc expression leading to G1 arrest and growth inhibition of human invasive urinary bladder cancer cells. Mol Cancer Ther 8: 458-468, 2009. 
17. Sulekova Z, Reina-Sanchez J and Ballhausen WG: Multiple APC messenger RNA isoforms encoding exon 15 short open reading frames are expressed in the context of a novel exon 10A-derived sequence. Int J Cancer 63: 435-441, 1995.

18. Zumbrunn J, Kinoshita K, Hyman AA and Nathke IS: Binding of the adenomatous polyposis coli protein to microtubules increases microtubule stability and is regulated by GSK3 beta phosphorylation. Curr Biol 11: 44-49, 2001.

19. Adamcikova Z, Wachsmannova L, Hainova K, Stevurkova V, Holec V, Ciernikova S, Cierna Z, Janega P, Babal P, Mego M, et al: Study of the APC gene function in the mouse APC+/APC1638N model. Neuro Endocrinol Lett 33: 26-33, 2012.

20. Liu XR, Shan XN, Friedl W, Uhlhaas S, Propping P and Wang YP: Analysis of germline mutations in the APC gene in familial adenomatous polyposis patients. Zhonghua Yi Xue Yi Chuan Xue Za Zhi 22: 261-264, 2005.

21. Pang CP, Fan DS, Keung JW, Baum L, Tang NL, Lau JW and Lam DS: Congenital hypertrophy of the retinal pigment epithelium and APC mutations in Chinese with familial adenomatous polyposis. Ophthalmologica 215: 408-411, 2001.

22. Cai SR, Zhang SZ and Zheng S: Detection of adenomatous polyposis coli gene mutations in 31 familial adenomatous polyposis families by using denaturing high performance liquid chromatography. Zhonghua Yi Xue Yi Chuan Xue Za Zhi 25 : 164-167, 2008 (In Chinese).

23. Zhang S, Qin H, Lv W, Luo S, Wang J, Fu C, Ma R, Shen Y, Chen S and Wu L: Novel and reported APC germline mutations in Chinese patients with familial adenomatous polyposis. Gene 577: 187-192, 2016.

24. Sheng JQ, Cui WJ, Fu L, Jin P, Han Y, Li SJ, Fan RY, Li AQ Zhang MZ and Li SR: APC gene mutations in Chinese familial adenomatous polyposis patients. World J Gastroenterol 16 $1522-1526,2010$

25. Jang YH, Lim SB, Kim MJ, Chung HJ, Yoo HW, Byeon JS, Myung SJ, Lee W, Chun S and Min WK: Three novel mutations of APC gene in Chinese patients with familial adenomatous polyposis. Cancer Genet Cytogenet 200: 34-39, 2010.

26. Song G, Yuan Y, Zheng F and Yang N. Novel insertion mutation p.Asp610GlyfsX23 in APC gene causes familial adenomatous polyposis in Chinese families. Gene 516: 204-208, 2013.

27. Chen S, Zhou J, Zhang X, Zhou X, Zhu M, Zhang Y, Ma G and Li J: Mutation analysis of the APC Gene in a Chinese FAP Pedigree with unusual Phenotype. ISRN Gastroenterol 2011: 909121, 2011

28. Li H, Zhang L, Jiang $\mathrm{Q}$, Shi $\mathrm{Z}$ and Tong $\mathrm{H}$ : Identification a nonsense mutation of APC gene in Chinese patients with familial adenomatous polyposis. Exp Ther Med 13: 1495-1499, 2017.
29. Wang TT, Chen SQ and Zhang XM: Germline mutation of adenomatous polyposis coli gene in Chinese patients with familial adenomatous polyposis. Zhonghua Yi Xue Yi Chuan Xue Za Zhi 25: 199-202, 2008.

30. Chen Q, Liu S, Feng J, Zhang X, Chen S, Ma G, Zhu M, Zhang Y and Yu J: Analysis of C.3925_3929 deletional mutations of APC gene in pedigrees with familial adenomatous polyposis. Zhonghua Yi Xue Yi Chuan Xue Za Zhi 32: 524-528, 2015 (In Chinese).

31. Chen QW, Zhang XM, Zhou JN, Zhou X, Ma GJ, Zhu M, Zhang YY, Yu J, Feng JF and Chen S: Analysis of small fragment deletions of the APC gene in chinese patients with familial adenomatous polyposis, a precancerous condition. Asian Pac J Cancer Prev 16: 4915-4920, 2015.

32. Zhang Z, Liang S, Wang D, Liang S, Li Y, Wang B, Jiang T, Zhao G, Zhang $X$ and Banerjee S: A novel pathogenic single nucleotide germline deletion in APC gene in a four generation Chinese family with familial adenomatous polyposis. Sci Rep 7: 12357, 2017.

33. Zhu Z, Huang J, Dong J, et al: Analysis of APC mutation in five kindreds of familial adenomatous polyposis. Med J West China 24: 1654-1657, 2012.

34. Liao DX, Li B, Du XM, Yu JH, Chang H, Wu ZQ, Hao HJ, Wang YX, Han WD, Cheng SJ and Luo CH: Two Chinese pedigrees for adenomatous polyposis coli: New mutations at codon 1309 and predisposition to phenotypic variations. Fam Cancer 13: 361-368, 2014.

35. Zhang J, Li Z, Huang X and Ye J: Clinical and molecular characteristics of a child with familial adenomatous polyposis. Zhonghua Er Ke Za Zhi 54: 205-208, 2016.

36. Pan H, Gao HL, Wu QY, et al: Diagnosis of APC gene mutation in a patient with familial adenomatous polyposis. Mil Med J Southeast China 16: 566-168, 2014.

37. Gan YB, Zheng $\mathrm{S}$ and Cai XH: Detection of a gene mutation in familial adenomatous polyposis families by PCR-RFLP method. Zhonghua Yi Xue Za Zhi 74: 352-354, 390-391, 1994 (In Chinese).

38. Zhang Z, Liang S, Huang H, Wang D, Zhang X, Wu J, Chen H, Wang Y, Rong T, Zhou Y and Banerjee S: A novel pathogenic large germline deletion in adenomatous polyposis coli gene in a Chinese family with familial adenomatous polyposis. Oncotarget 7: 50392-50400, 2016.

(7) $\Theta$ This work is licensed under a Creative Commons cc) Attribution-NonCommercial-NoDerivatives 4.0 International (CC BY-NC-ND 4.0) License. 